\title{
Le rôle des périodiques savants dans la diffusion des savoirs géographiques pendant la Révolution française
}

Isabelle Laboulais-Lesage

\section{(2) OpenEdition Journals}

Édition électronique

URL : https://journals.openedition.org/ahrf/1584

DOI : 10.4000/ahrf.1584

ISSN : 1952-403X

Éditeur :

Armand Colin, Société des études robespierristes

Édition imprimée

Date de publication : 1 décembre 2004

Pagination : 53-74

ISSN : 0003-4436

\section{Référence électronique}

Isabelle Laboulais-Lesage, « Le rôle des périodiques savants dans la diffusion des savoirs géographiques pendant la Révolution française ", Annales historiques de la Révolution française [En ligne], 338 | octobre-décembre 2004, mis en ligne le 15 décembre 2007, consulté le 23 avril 2022. URL : http://journals.openedition.org/ahrf/1584 ; DOI : https://doi.org/10.4000/ahrf.1584

Ce document a été généré automatiquement le 23 avril 2022

Tous droits réservés 


\title{
Le rôle des périodiques savants dans la diffusion des savoirs géographiques pendant la Révolution française
}

\author{
Isabelle Laboulais-Lesage
}

À la fin du XVIIIe siècle, faire de la géographie consiste à proposer une description exhaustive de la terre. Cette vaste ambition conduit non seulement à une subdivision de ce champ en plusieurs catégories -la géographie ancienne, la géographie du Moyen Age, la géographie moderne, la géographie physique, la géographie astronomique, la géographie civile, la géographie politique, etc.-, mais l'ampleur de l'objet complique également son positionnement institutionnel ${ }^{1}$. Rappelons pour mémoire que jusqu'en 1793, il n'existe à l'Académie royale des Sciences aucune classe de géographie, ce domaine de savoir est simplement représenté par une charge d'adjoint géographe. De plus, la géographie ancienne est également étudiée à l'Académie des Inscriptions et Belles Lettres ${ }^{2}$. Ce manque de visibilité institutionnelle contraste pourtant avec le rôle fondamental joué par la géographie dans la culture de l'honnête homme. Ce savoir descriptif, immédiatement accessible à n'importe quel lecteur, invite au dépaysement, à la connaissance des espaces lointains et fait donc de la géographie une science aimable, une science qui dispose à la fois d'un public considérable et d'un large vivier d'auteurs. En effet, à l'exception de la production cartographique qui nécessite des compétences spécifiques, la production du savoir géographique n'exige aucun savoirfaire particulier. La rédaction d'une description n'est régie par aucune contrainte de méthode ; elle ne fait l'objet d'aucune codification, ni de la mise en place d'aucune langue spécifique. Cette caractéristique explique la facilité et la rapidité avec lesquelles des ouvrages de géographie sont rédigés par des publicistes qui ne jouissent d'aucune réputation de géographe. D'ailleurs, si sous l'Ancien Régime, quelques-uns peuvent être identifiés grâce à leur titre de «Géographe du roi », il reste illusoire de chercher à retrouver des critères qui permettraient de reconnaître des géographes «professionnels ». De même que la géographie s'adresse à un public mondain plus qu'à 
un public savant, elle est du ressort de "géographes autoproclamés » qui, à l'image de Pluche ou de Nollet, ne sont pas des savants de premier plan.

2 Au cours des années 1790, la géographie conserve son statut de science aimable ; différents supports sont alors mobilisés pour transmettre les savoirs de l'espace et promouvoir la pédagogie du territoire. Les trois dimensions de la vulgarisation -l'écrit, la parole, l'image ${ }^{3}$ - sont utilisées: des cours et des conférences publiques de géographie sont dispensées à Paris et dans les grandes villes de province, des manuels et des traités de géographies, de même que plusieurs méthodes d'enseignement consacrées exclusivement à la géographie sont publiés, des cartes et des atlas sont éditées, des jardins géographiques sont imaginés ${ }^{4}$, enfin des promenades pédagogiques sont organisées. Les périodiques savants offrent un poste d'observation approprié pour mesurer à la fois la diversité des supports convoqués pour la promotion des savoirs géographiques et pour évaluer leurs conditions de réception. Si certains périodiques se contentent d'annoncer les ouvrages publiés, d'autres, au contraire, ne manquent pas d'exprimer des critiques à l'encontre des auteurs et de leurs compétences. De manière à croiser des points de vue différents, nous avons retenu quatre périodiques généralistes : le Bulletin de la littérature, des sciences et des arts, le Bulletin des sciences de la Société philomathique de Paris, la Décade philosophique littéraire et politique, le Magasin encyclopédique ou Journal des sciences, des lettres et des arts et trois périodiques spécialisés : le Journal des Mines, le Journal des Arts et manufactures, et les Annales de statistique ou Journal général d'économie politique (...), de géographie ${ }^{5}$. Parmi ces trois derniers titres, aucun n'est spécifiquement consacré à la géographie mais tous s'inscrivent dans des domaines de savoir qui ont pour objet la connaissance et la compréhension d'agencements spatiaux : la géologie et la statistique. À partir de cet échantillon, nous chercherons à montrer si les périodiques savants de la Révolution ont contribué à donner de la visibilité aux savoirs géographiques et nous nous demanderons quelle position ils leur ont conféré tant dans le monde savant que dans la société régénérée. Il semble en effet que le schéma classique qui consiste à repérer pendant la Révolution le passage d'une "science mondaine " à une "science sévère » ne corresponde pas aux évolutions survenues dans le champ des savoirs géographiques. Nous nous demanderons au contraire si, à cette période, les anecdotes de la science mondaine ne cèdent pas progressivement du terrain devant les exigences de la science utile. Nous verrons de quelle manière les périodiques savants traduisent ce nouveau rapport au territoire caractéristique de la période révolutionnaire et mis en lumière par des nombreux travaux ${ }^{6}$, nous observerons comment ils traduisent la consubstantialité du territoire et de la Nation ainsi que l'interdépendance de la connaissance spatiale et de la mise en œuvre de l'action publique. Pour cela, nous nous attacherons tout d'abord à la place laissée aux textes géographiques dans les périodiques savants de la Révolution, puis nous analyserons l'image que les comptes rendus donnent de la production géographique, enfin nous verrons comment certains titres se présentent comme des acteurs du renouvellement des savoirs géographiques.

I. Diffuser les savoirs géographiques

3 Les périodiques savants publiés pendant la Révolution française conservent une forme semblable à celle fixée dès l'Ancien Régime ${ }^{7}$; ils sont composés d'articles assez substantiels, de comptes rendus plus ou moins développés et d'annonces plus brèves. Tous ces textes sont répartis par genre, puis, le plus souvent, sont classés en rubriques thématiques. Or, comparés aux nombreux articles consacrés à l'histoire naturelle, à la 
physique, à la chimie, ou à la médecine, ceux qui paraissent dans une rubrique " géographie » occupent une place infime dans les périodiques que nous avons retenus. Ainsi, entre 1791 et 1805, seules quatre contributions du Bulletin des sciences de la Société philomathique sont publiées dans une rubrique explicitement consacrée à la géographie, et encore pour deux d'entre eux l'intitulé exact est : « géographie physique ». Les deux premiers rassemblent des considérations sur la cartographie, les deux autres des observations de voyage faites respectivement par Girod de Chantrans et Humboldt ${ }^{8}$. Dans la Décade philosophique, entre floréal an II et prairial an VI, un seul article est proposé dans une rubrique "géographie", il est intitulé : "Des possessions que la République française acquiert par le traité de Campo-Formio sur les côtes d'Albanie et la Mer Ionienne "; l'ambition de son auteur consiste à " examiner successivement la situation et l'importance de ces ports et de ces îles sous les rapports de la population, de la Marine et du Commerce, etc. » ${ }^{9}$. Enfin, dans le Magasin encyclopédique, entre 1795 et 1799 , six contributions paraissent dans une rubrique nommée " géographie ", quatre s'attachent à la géographie moderne alors que les deux autres relèvent de la géographie ancienne ${ }^{10}$.

4 Parmi ces onze textes, on dénombre quatre notices descriptives qui rendent compte d'espaces peu connus, deux contributions érudites qui s'attachent à la géographie de l'antiquité, deux observations de voyage reliées à la géographie physique, deux séries de considérations sur la construction des cartes, et un texte méthodologique consacré à l'enseignement de la géographie. Cet ensemble reflète bien l'ampleur du domaine qu'entend embrasser la géographie. Pourtant, l'ambition de cette science qui transmet un savoir spatial sur le monde présent et passé contraste avec sa présence modeste dans les périodiques savants. Ces imprimés confirment le manque de visibilité de la géographie, et peut-être aussi le manque de dynamisme suggéré par Delambre, dans les Rapports à l'Empereur ; il estime en effet que la France a laissé aux Anglais « le flambeau de la recherche géographique ${ }^{11}$.

Cette approche nominaliste qui consiste à ne prendre en compte que les articles publiés dans une rubrique "géographie " ne peut cependant suffire à saisir la présence de savoirs géographiques dans les périodiques savants, a fortiori quand on songe qu'à cette époque la synonymie entre voyage et géographie semble aller de soi. Les récits de voyage sont en effet considérés comme une source de données qui contribuent de manière essentielle à la connaissance de la surface du globe. Pourtant, en dépit de l'engouement suscité à cette époque par les voyages, assez peu d'articles publiés dans les périodiques savants évoquent les manières de voyager ${ }^{12}$. Certes, il existe une rubrique consacrée aux voyages dans la Décade philosophique et dans le Magasin encyclopédique mais les comptes rendus y sont bien plus nombreux que les articles. Ceux-ci ressemblent le plus souvent à des narrations anecdotiques, à l'image des extraits de lettres ou de carnets de voyage inédits que publie la Décade philosophique ${ }^{13}$. Il ne s'agit que très rarement d'analyses circonstanciées. Ainsi, lorsqu'en 1796, la Décade philosophique et le Magasin encyclopédique reproduisent l'exposé présenté par la Billardière à la première classe de l'Institut pour rendre compte des apports de son voyage à la recherche de Lapérouse ${ }^{14}$, ce texte paraitt très isolé. Les périodiques savants rendent compte des récits de voyage publiés, nous y reviendrons, mais ils ne s'intéresse pas à « la politique des voyages » développée à cette période ${ }^{15}$. Un extrait de la préface du premier numéro des Annales de statistiques offre une explication à ce silence: 
l'auteur condamne en effet la préférence trop souvent accordée par les lecteurs de géographie à l'évocation de terres lointaines :

«Car, si nous lisons avec empressement les voyages de Cook, Lapeyrouse, des Vaillant, qui nous promènent dans des contrées agrestes et sauvages, où végètent des peuples barbares, lirons-nous avec moins d'intérêt l'ouvrage destiné à nous faire connaître le plus beau, le plus curieux de l'univers, le pays des découvertes, des arts et de l'industrie, habité par un peuple aimable et poli, autant que brave et éclairé [...]. Notre pays, enfin? Ce genre d'étude a véritablement une utilité réelle, en ce qu'elle nous fait connaitre les ressources, les institutions, les usages, les mœurs des peuples; connaissance qui mène à celle du cœur humain et peut nous affranchir de ces préjugés locaux qui ont fait si souvent plusieurs peuples d'une même nation.» ${ }^{16}$

Les voyages lointains sont rattachés à la culture mondaine et semblent désormais plus futiles au regard des descriptions du territoire national. À l'image des Annales de statistiques, tous les périodiques spécialisés de notre corpus s'efforcent de répandre un usage fécond de la géographie. Celle-ci n'y est jamais regardée comme un savoir spéculatif mais au contraire comme un savoir indispensable aux hommes de la pratique. Dans le Journal des mines et les Annales de statistiques, la géographie quitte résolument l'horizon de la culture mondaine pour être mise au service de la minéralogie et de la statistique. La réalisation de la «description minéralogique de la France » le montre bien. Ce projet est annoncé dès le premier numéro du Journal des mines et la parution de ce qui est finalement désigné comme le «Tableau des mines et usines de la République » commence à partir du numéro 23 avec une notice consacrée au département de l'Ain. Toutes les descriptions publiées dans le Journal des Mines proposent d'abord une "notice géographique» du département concerné. Cette première partie permet de présenter les caractéristiques physiques du territoire décrit (limites et étendue, montagnes, rivières), l'histoire du lieu, la population, la nature du sol, les cultures, l'industrie et le commerce. Dans une note de bas de page de la description des Basses-Alpes, Coquebert explique la raison d'être de ces notices géographiques :

"Ces rapides aperçus de la géographie physique, historique et commerciale de chaque département, ne sont point étrangers au but que le conseil des mines s'est proposé en publiant ce travail. Nous cherchons à y réunir en peu de mots ce que tout voyageur, quel que soit l'objet particulier de ses recherches, a besoin de savoir d'une manière générale touchant le département qu'il se propose de visiter.

D'ailleurs, parmi les circonstances qui font prospérer les mines, ou qui s'opposent à leur succès, il faut compter, sans doute, l'abondance ou la rareté des subsistances, une population nombreuse ou faible des branches d'industrie qui puissent s'associer aux travaux des mines ou qui leur soient contraires, l'état plus ou moins florissant des lieux de consommation, celui des routes, des rivières, des canaux, \&c. Ces éléments doivent entrer dans le calcul de quiconque se propose d'entreprendre ou de suivre des exploitations: ils sont également nécessaires à ceux que le gouvernement charge de traiter, sous le rapport de l'intérêt public et particulier, des questions relatives aux mines. " "

7 Ces notices désignées comme "géographiques » permettent, d'une part, d'ancrer les données liées aux mines dans un espace précis, d'autre part, elles montrent que la connaissance des ressources naturelles et des activités humaines est nécessaire à tout projet d'inventaire. Les ambitions de cette description minéralogique de la France ne sont pas purement spéculatives ; cette enquête doit au contraire conduire à l'amélioration de l'exploitation des richesses et pour cela, la connaissance des « circonstances " géographiques est indispensable. Ces notices publiées dans le Journal 
des mines présentent une grande similitude avec le tableau présenté dans les Annales de statistiques pour servir de cadre à toutes les topographies départementales. Dans ce tableau sont évoquées les rivières principales, les montagnes, les vallées...etc. Le milieu géographique constitue donc le cadre obligé de toute description des territoires. Qu'il s'agisse de minéralogie ou de statistique, la géographie doit leur fournir les cadres capables d'agencer les données concernant un domaine d'activité spécifique, en l'occurrence ici les mines, ou bien les rouages économiques et sociaux. La géographie se trouve souvent réduite à une simple nomenclature des lieux ; d'ailleurs le mot topographie se substitue parfois au mot géographie. Chaptal expose par exemple le plan de son enquête en affirmant que « la topographie est le cadre dans lequel viennent se placer les faits et les notions $»{ }^{18}$.

8 Le Journal des arts et manufactures propose quant à lui une approche singulière de la géographie, il ne s'attache en effet qu'à l'un de ses aspects et promeut la "géographie industrielle ». Ce domaine de savoir est désigné comme " la reunion des connoissances relatives au nombre, à l'importance \& à la distribution sur le territoire des établissemens industriels, tels que manufactures, fabriques, \&c. ${ }^{19}$. Cette définition montre bien que la géographie n'est pas étudiée pour elle-même. Ce périodique se charge en effet de publier les résultats de l'enquête organisée par la commission d'agriculture, arts et manufactures pour obtenir une statistique sommaire de l'équipement industriel par produit et par lieu ${ }^{20}$. La géographie est donc unanimement regardée comme une science des lieux. Dans les périodiques généralistes, elle apparaît comme un élément mineur de la culture savante; dans les périodiques spécialisés, elle devient une sorte de préalable pour des disciplines qui cherchent à rendre compte d'une forme spatiale ${ }^{21}$. Les périodiques savants offrent donc un reflet fidèle du statut épistémologique complexe qui est celui de la géographie au tournant du XVIIIe et du XIXe siècle : la connaissance géographique est jugée indispensable, mais son ambition de décrire la terre paraît si vaste que la géographie se trouve fractionnée entre plusieurs champs et manque ainsi de visibilité.

II. Dessiner en creux les attentes d'une géographie révolutionnée

9 Depuis l'Ancien Régime, les périodiques savants publient des comptes rendus d'ouvrages. Certains même y consacrent toutes leurs pages, c'est le cas notamment du Bulletin de la littérature, des sciences et des arts. Les comptes rendus côtoient souvent les annonces. Celles-ci ramenées le plus souvent à la fin des périodiques se contentent de signaler les parutions récentes, sans en proposer aucun commentaire, elles signalent également les cours ou les conférences. Les cours de géographie dispensés par Mentelle sont ainsi annoncés dans plusieurs périodiques. Dans la Décade, c'est à l'occasion de la parution de la carte des Pays-Bas par Mentelle que les rédacteurs signalent l'ouverture de son cours :

«Nous saisissons cette occasion pour annoncer que le citoyen Mentelle a ouvert primedi dernier, son cour de géographie. On connaît l'excellente méthode de ce géographe : il suit une marche que la Nature a indiquée ; il vous montre d'abord le globe terrestre sous sa forme physique, et sans aucune division, ni constructions humaines. On y distingue les formes des terres, les montagnes, les mers, les rivières. Il trace ensuite sur ce globe les divisions que les anciens avaient faites, les cités qu'ils avaient bâties, et les remplace enfin par les divisions actuelles. On souscrit chez lui, cour carrée du Muséum, pour ce cours qui se continue les jours impairs de chaque décade, et qui est du prix de 30 livres. Il y a douze places gratuites à la nomination de la commission d'Instruction publique.» ${ }^{22}$ 
10 Deux ans plus tard, sur la troisième de couverture du $n^{\circ} 74$ de la Décade philosophique, les «cours réunis de géographie et d'histoire, de sciences sociales, de langue et littérature anglaise, de langue et littérature italienne » dispensés par les citoyens Mentelle, Perreau, Robert et Boldoni sont annoncés ${ }^{23}$, on retrouve cette même information dans le Magasin Encyclopédique ${ }^{24}$. Néanmoins, pour saisir l'opinion des rédacteurs des périodiques sur la géographie, c'est plutôt vers les comptes rendus qu'il faut se tourner. Certes, tous ne proposent pas une lecture critique des ouvrages ; quelques-uns se contentent même de reproduire de longs extraits des livres concernés[2020 25 . Toutefois, quelques textes plus substantiels permettent de mesurer ce qui est attendu de la géographie et le statut qui est conféré aux savoirs géographiques pendant la Révolution; ils concernent principalement des récits de voyages ${ }^{26}$, quelques cartes, mais aussi des manuels de vulgarisation. Si les pratiques de voyage ne donnent lieu qu'à très peu d'articles, la littérature de voyage en revanche suscite de nombreux comptes rendus. Les rédacteurs des périodiques savants la regardent comme un moyen de connaître le monde et les «variétés de l'étonnante espèce humaine " ${ }^{27}$, mais les informations que ces livres recèlent peuvent aussi contribuer à développer le commerce c'est-à-dire à promouvoir la puissance de la nation. Au début du compte rendu du récit de voyage de Forster, on peut lire :

«L'histoire des voyages est une branche nourricière des arts et des sciences. C'est par elle que nous obtenons chaque jour des résultats précieux pour l'humanité, qu'un pays s'enrichit des productions d'un autre, et s'approprie tout ce qui peut lui être utile. ${ }^{28}$

11 Un des rédacteurs de la Décade va même jusqu'à regarder la traduction française du voyage de J. Long en Amérique du Nord comme " une espèce de prise sur nos ennemis » ${ }^{29}$. Les données géographiques paraissent si précieuses que, même lorsque la qualité des récits de voyage est jugée moyenne, les rédacteurs en recommandent néanmoins la lecture. Ainsi, dans le compte rendu de la Relation de l'ambassade du Lord Macarteney à la Chine dans les années 1792, 1793 et 1794, l'auteur insiste sur les informations consacrées au peuple chinois et, même s'il considère que les observations faites pendant ce voyage sont superficielles, face à l'absence de connaissances générales sur la Chine, il conseille de consulter ce récit ${ }^{30}$. La diversité géographique des voyages publiés doit permettre aux lecteurs de se doter d'une culture universelle ; sur ce point, les rédacteurs sont unanimes. Cette abondance suscite pourtant quelques réticences de la part de certains, plusieurs adressent ainsi de vives critiques aux traducteurs peu expérimentés et aux éditeurs peu scrupuleux qui livrent au public des ouvrages de mauvaise qualité :

« Nos libraires s'empressent de publier en français un ouvrage dès qu'il jouit de quelque succès dans l'étranger ; pour vendre le livre, ils comptent sur son titre plutôt que sur son mérite ; ils visent à faire promptement plutôt qu'à faire bien ; ils s'adressent à des littérateurs de peu de capacité, souvent à plusieurs traducteurs pour achever plutôt [sic] un même ouvrage, et c'est ainsi que nous voyons paraître et des voyages et des romans et des livres de morale et des livres d'agriculture, traduits par des personnes qui ne se doutent pas qu'il faut pour bien traduire posséder deux qualités indispensables. La première est d'entendre la langue qu'on traduit ; c'est-à-dire d'entendre jusqu'à ses finesses, et l'on n'y parvient qu'après avoir vécu dans le pays où on la parle. Ensuite il faut savoir écrire dans sa propre langue, et cette dernière qualité n'est pas la plus commune, ni la plus facile à acquérir. ${ }^{31}$

12 Cette critique ne vaut évidemment pas seulement pour les récits de voyage ; néanmoins le succès qu'a connu ce genre d'ouvrages au cours de cette période et la dimension 
plaisante de cette littérature rend la critique particulièrement pertinente. On voit ici poindre l'importance conférée aux savoirs géographiques: dans la mesure où ils sont considérés comme utiles à l'essor de la puissance nationale, il ne faut pas que les lecteurs puissent être abusés par des ouvrages qui dispenseraient des informations erronées. On mesure combien les niveaux d'exigence de la science mondaine et de la science utile sont distincts. Ce souci de précision transparait également lorsque les compétences des voyageurs sont analysées. Dans le Magasin encyclopédique, Faujas de Saint-Fond est par exemple présenté comme un naturaliste distingué et comme un très bon observateur ${ }^{32}$. Mais, au-delà de ces appréciations individuelles, on trouve aussi dans certains comptes rendus des opinions plus générales. Ainsi, dans la Décade philosophique, l'auteur qui évoque le Voyage philosophique et pittoresque sur les rives du Rhin de Georg Forster s'éloigne un temps de son propos pour consacrer un paragraphe entier à décrire ce qu'il considère comme la meilleure méthode pour rédiger un récit de voyage :

«La méthode de Forster est celle qu'ont suivie plusieurs voyageurs modernes avec plus ou moins de succès. Il ne se borne pas à décrire ce qu'il voit, ni à rédiger ce qu'il observe ; les sentiments qu'il éprouve, les méditations philosophiques ou politiques auxquelles il se livre, les digressions sur les arts et leurs principes, sur les événements et leurs causes, sur la nature et ses étonnants phénomènes, l'occupent, l'arrêtent, et obligent le lecteur à s'arrêter pour ainsi dire, à chaque pas comme lui. Cette méthode a ses avantages; elle peut avoir aussi ses abus. Un auteur qui, à propos de tout ce qu'il voit, dit tout ce qu'il sent et tout ce qu'il sait, met facilement dans son ouvrage un désordre fatigant, une plénitude surabondante, une sorte d'agitation convulsive, qui lasse, rassasie et trouble l'esprit. Il éteint l'intérêt à force d'en multiplier les moyens; à force d'entasser sans mesure les sensations et les idées, les observations et les discussions, les mouvemens de l'âme et les recherches de la science, il neutralise les uns par les autres; et ceux qui s'engagent avec lui dans cette espèce de labyrinthe, après tant de tous et de détours, après une course longue et souvent pénible, se retrouvent enfin, comme dans celui de Crète, au même point dont ils étoient partis. » ${ }^{33}$

On retrouve ici l'attachement exprimé pendant la Révolution à la production d'un savoir utile et objectif ou "exact». Désormais, les appréciations personnelles paraissent accessoires, voire nuisibles à la qualité de l'ouvrage et c'est à l'aune des besoins de la nation que la qualité d'un récit est évaluée. Chaque voyageur doit donc diffuser des observations précises de manière à contribuer au renouvellement du savoir géographique. De manière implicite, on retrouve ici une argumentation semblable à celle qui justifie le recours aux instructions de voyage. D'ailleurs, la plupart des périodiques consultés dans cette recherche signalent ou rendent compte en 1797 de la traduction par C.-P. de Lasteyrie de l'ouvrage de Berchtold, Essai pour diriger et étendre les recherches des voyageurs qui se proposent l'utilité de leur patrie. L'utilité de la patrie apparaît aussi comme l'un des critères qui président à l'évaluation de la production cartographique. Coquebert en témoigne dans le Magasin encyclopédique à propos des cartes de Suède publiées par Hermelin qu'il considère comme « une nouvelle preuve de cet esprit public qui fait tant d'honneur à la Suède ${ }^{34}$. Il commence ainsi sa recension :

«Entre plusieurs autres qualités excellentes, les Suédois ont celle de préférer leur pays à tous les autres, bien différents en cela de tant d'hommes prévenus, frondeurs ou ingrats, à qui rien ne peut plaire s'il n'est étranger. Les savans de cette nation se sont appliquées à bien connaître leur patrie, et à la faire connoître avantageusement aux autres peuples : veilles, recherches, dépenses même, rien ne leur a coûté pour remplir cette tâche estimable. " ${ }^{35}$ 

doivent contribuer à dire la grandeur de la Nation et travailler à l'amélioration des aménagements du territoire. Ce même aspect retient l'attention du rédacteur du Magasin encyclopédique lorsqu'il rend compte de la Carte de la France où l'on a essayé d'exprimer les configurations de son territoire, par une nouvelle méthode de nivellement, carte réalisée par l'ingénieur géographe Dupain-Triel ; il souligne en effet :

"Ce travail a été entrepris pour donner une première idée des effets d'un résultat plus exact, qu'on obtiendra par des nivellemens faits pour le grand objet de compléter la géographie physique de la France, et qui conduiront à la connoissance des communications qu'on peut établir sur le territoire françois, en même temps qu'on y étudiera le meilleur emploi des eaux pour la navigation, l'agriculture, les arts et la défense de nos frontières. " ${ }^{36}$

Les mesures faites sur le terrain permettent de produire une carte précise, celle-ci constitue un outil précieux pour les administrateurs. L'utilité des cartes n'est pas seulement évaluée du point de vue des usages politiques, elle l'est aussi à l'aune de leur utilité pour le citoyen. La bonne connaissance du territoire que l'on est en droit d'attendre de lui suppose en effet qu'il puisse se le représenter mentalement et, pour cela, la consultation des cartes parait nécessaire. Certains, à l'image de l'auteur qui rend compte de l'Atlas méthodique et élémentaire de géographie et d'histoire publié chez Desnos, recommandent l'utilisation de cartes simplifiées pour faciliter l'apprentissage de la géographie :

"L'expérience a prouvé qu'avec cet atlas, les pères, les mères et les maitres les moins instruits, pouvaient enseigner les différentes sciences, qui en sont l'objet ; que même les secours d'un maître n'étaient pas nécessaires; les planches offrant aux yeux ce que le discours enseigne à l'esprit ; elles en rendent le sens plus facile à entendre ; et comme les figures et les cartes qu'elles présentent, se peignent dans la mémoire et que le souvenir s'en conserve mieux que celui du discours, elles rappellent ce qu'il contient, l'étude, rendue plus facile par ces planches devint aussi par elles bien moins fatigante et bien plus prompte. ${ }^{37}$

On retrouve ici le rôle essentiel de la géographie qui consiste alors à donner à voir le territoire, à mettre sous les yeux des lecteurs ce qui est absent, ce dont ils n'ont pas une conscience immédiate et qu'ils doivent cependant connaitre. Dans le Bulletin de la littérature, des sciences et des arts, les comptes rendus relatifs à la production cartographique donnent souvent aux auteurs l'occasion de revenir sur les transformations et les conquêtes de la République. Voici par exemple le début de la recension de la carte de Raymond consacrée au département du Mont-Blanc:

«Le vœu de ce département pour sa réunion à la République française n'a été qu'un cri unanime ; c'est en vain que le despotisme s'efforça pendant dix siècles, de séparer ce peuple de la France, pour le réunir à la nation piémontaise ; les barrières que la nature avait placées entre eux, la haine et l'antipathie ont toujours contrarié ces liens factices. Les habitants de la ci-devant Savoye ont constamment retenu le langage, les mœurs et les habitudes des Français, et lorsque le drapeau tricolor [sic] a flotté sur leurs montagnes, ce n'est point une nation étrangère que la république a conquise, ce sont des anciens Français dont elle a brisé les fers pour les réunir à leur famille primitive. ${ }^{38}$

17 Dans ces comptes rendus, ce ne sont pas les considérations cartographiques qui priment, ni même l'argument de l'exactitude, mais la volonté de légitimer les transformations territoriales et le souci de les faire connaître. De telles préoccupations apparaissent également dans les nombreux ouvrages de vulgarisation publiés pendant la Révolution et destinés aux élèves et aux citoyens. Dès le début des années 1790, de 
nombreux traités de géographie paraissent avec l'objectif de faire connaitre les nouveaux maillages territoriaux et de transmettre les changements de toponymes ${ }^{39}$. Cependant, l'absence d'une communauté de géographes identifiés par des critères de légitimité stables permet à tous les plumitifs de rédiger ce type de traités et, à de nombreuses reprises, les rédacteurs des périodiques savants signalent que ces auteurs n'accordent pas tout le soin nécessaire à leurs travaux. Ainsi dans le compte rendu de la Géographie élémentaire de Sérane, le rédacteur du Magasin encyclopédique note :

«Dans un livre élémentaire on ne cherchera point des découvertes importantes, mais du moins on doit s'attendre à y trouver ce qui est connu, présenté avec méthode, et surtout de ne point $\mathrm{y}$ rencontrer d'erreurs grossières. Malheureusement, en parcourant ce petit volume, nous y avons rencontré une foule d'inexactitudes et de fautes d'impression d'autant plus fâcheuses, que c'est un livre destiné à l'instruction de la jeunesse, à laquelle il est tout aussi facile de dire les choses telles qu'elles sont, que de ne pas le faire. " ${ }^{40}$

18 Cet ouvrage paraît représentatif de la mauvaise vulgarisation : la volonté de mettre un savoir à la portée de chacun ne peut en effet justifier la présence d'aucune approximation. Or, l'auteur du compte rendu signale que ce livre recèle de nombreuses erreurs et il insiste sur les dangers de celle-ci. Les reproches adressés aux géographes de procéder à une compilation aveugle et de ne pas vérifier leurs sources sont des arguments classiques, largement utilisés aux XVIIe et XVIIIe siècles. Ici pourtant, ils prennent plus d'ampleur en raison de la finalité de ces géographies. Elles doivent servir à la formation du citoyen et celle-ci ne peut être prise à la légère, ce que les géographes semblent faire trop souvent. L'un des rédacteurs de la Décade philosophique les présente à la fois comme de faux savants et comme des plumitifs soumis aux exigences des monarques :

« Des compilateurs sans goût, des copistes à gages, des écrivains mercenaires vous traînoient à leur suite de provinces en provinces, tout couvertes de la poudre des anciens titres, et des restes dégoûtants de l'histoire des premiers siècle de la monarchie. Pleins de vénération pour tous les mensonges ecclésiastiques, et de respect pour nos premiers tyrans, ils vous racontoient tranquillement tous les forfaits qui leur avoient acquis tels ou tels lambeaux de la France; puis il vous conduisoient de châteaux en châteaux, d'abbayes en prieurés, vous étaloient tous les titres des gouverneurs, tous les privilèges et les richesses des moines et autres objets de la même importance. S'ils parloient du peuple, de son industrie, de sa richesse, ce n'étoit qu'autant que ces objets pouvoient entrer dans le calcul des revenus du prince. Ils ne voyoient que lui partout ; et quand ces malheureux avoient traité longuement du roi de France, des grands officiers de la couronne, et de tous les inutiles de l'Etat, ils croyoient n'avoir plus rien d'important à vous apprendre. En vous donnant la description de la France, ils n'avoient rien oublié... que la Nation. ${ }^{41}$

19 Certes, ce sont ici les géographes d'Ancien Régime qui sont visés, cependant quelques mois plus tard, un rédacteur de la Décade affirme que la tutelle exercée par le gouvernement sur les géographes n'a pas disparu avec la Révolution :

«Les changemens de noms de tant de lieux ont éprouvé depuis notre révolution, ont désolé les géographes. Les plus sages ont attendu et attendent encore patiemment que tout ait pris son assiette ; car ils ne veulent point faire la géographie de l'assemblée constituante, ni celle de Robespierre, ni celle de Marat, mais celle de la République française. Espérons que bientôt nous ne verrons plus nos livres et nos cartes géographiques souillés par des dénominations qui attestent la barbarie et la stupidité de leurs parrains. ${ }^{42}$ 
La Géographie de France, suivant la division en 88 départemens semble pourtant avoir réussi à gommer les préjugés anciens, et avoir rendu aux considérations géographiques ce qui est regardé comme une forme d'objectivité :

« son livre est un des plus intéressans de ce genre qui aient paru depuis la nouvelle division de la France, et qu'il parait purgé de ces nombreuses sottises dont nos précédentes géographies sont remplies: sottises religieuses, sottises politiques, sottises historiques, préjugés de nations, préjugés d'états, préjugés de physique, etc. toutes choses qu'on fesait effrontément apprendre aux malheureux enfants, comme de belles et bonnes vérités. » ${ }^{43}$

21 Outre qu'elle est débarrassée des préjugés, cette Géographie de France semble parvenir aussi à éviter les deux travers que les savants prêtent traditionnellement à la géographie : proposer des nomenclatures trop arides qui finalement rebutent le lecteur et se contenter de descriptions anecdotiques, guidées par l'obsession de plaire, voire de distraire aux dépens de l'exactitude. Ce livre est à la fois présenté comme débarrassé de toute monotonie et agréable à lire :

«On voit que l'auteur de cette géographie n'a rien négligé pour égayer l'aridité de la matière qui ordinairement ne présente que des descriptions sèches qui rebutent les jeunes-gens, bien loin de leur inspirer du goût pour l'étude d'une science si intéressante et nécessaire à connaître. " ${ }^{44}$

Cependant le plaisir de la lecture n'efface pas la qualité des informations contenues dans l'ouvrage :

«Il donne d'abord, sur chaque département, quelques notions générales, puis il passe en revue chaque ville, bourg, etc., en rapportant les traits historiques, les coutumes que ces lieux rappellent, les branches d'industrie qu'on y cultive, le costume de leurs habitants, les noms des personnages illustres qui y ont pris naissance. Il décrit les curiosités naturelles, les monumens anciens, gothiques, ou modernes, qui s'y trouvent, et ne perd pas l'occasion de raconter une anecdote ou de faire une citation. »

Le rédacteur de la Décade ajoute quelques pages plus loin que l'auteur ne néglige aucun aspect de la description et s'efforce de donner un aperçu des lieux aussi fidèle que possible :

«Il est trop ordinaire de ne trouver dans les géographies que des descriptions de villes et de bourgs : on y cherche et l'on y trouve rarement les descriptions du pays, des campagnes et lorsqu'il arrive qu'on voyage sur les lieux, on s'aperçoit qu'on ne les connaitt point. Leur aspect quelquefois si imposant, d'autres fois si riant et si doux, vous était absolument inconnu après les avoir étudiés dans les livres et sur la carte. L'auteur de la Géographie de France semble avoir voulu éviter ce reproche ; et quoique son style ne réponde pas toujours à son sujet, et qu'il soit en général trop peu travaillé, on aime à trouver dans son livre des descriptions comme celle-ci, que nous prenons au hasard. » ${ }^{45}$

24 Une bonne géographie doit donc être agréable à lire, fidèle et exhaustive. Ce sont ces mêmes qualités qui sont aussi prêtées à l'ouvrage de Lavallée et Brion, le Voyage dans les départements de la France ${ }^{46}$. Dans le Bulletin de la littérature, des sciences et des arts, il est présenté comme " absolument neuf »; et l'auteur donne ensuite les caractéristiques de ce qui est, à ses yeux, une bonne géographie "régénérée » :

«C'est une galerie pittoresque et descriptive, où l'observateur voit la France telle qu'elle est, et où il saisit d'un coup d'œil tous les rapports et toutes les nuances d'intérêts et de caractères qui existent entre les habitants de cette vaste république. L'exacte topographie et les faits les plus remarquables de l'histoire de chaque département et de chaque ville ; la description des monuments antiques et 
modernes que le temps et le vandalisme ont épargné ; des anecdotes piquantes sur les progrès des arts et des sciences ; des notions instructives sur les mœurs des habitants des diverses contrées que le voyageur parcourt; des digressions intéressantes mêlées de réflexions philosophiques et de quelques données sur le gouvernement donnent à cet ouvrage le plus grand intérêt. Les planches dont il est enrichi, sont artistement gravées et imprimées sur un très beau papier. Le style de ce voyage nous a paru un peu trop recherché et même quelquefois ampoulé. [...] Malgré cette légère imperfection, on lira avec le plus grand plaisir ce voyage qui est fait pour intéresser tous les physiciens, les géographes, les politiques et les littérateurs. » ${ }^{47}$ trouve ici concilié avec le souci de précision, et la volonté de s'instruire est mêlée au plaisir de lire. L'influence de la cosmographie descriptive héritée de la Renaissance -et particulièrement l'ambition universaliste de la description- reste prégnante dans les critères d'évaluation des géographies. Cependant, lorsqu'il s'agit d'évaluer la qualité des géographies de la France destinées à un large public, ces attentes traditionnelles de la science mondaine sont croisées avec les exigences de la science utile. Pourtant, aux yeux des rédacteurs de la Décade, ces critères ne suffisent pas à légitimer la présence d'un cours de géographie à l'École normale de l'an III. Lorsqu'il est question de l'enseignement de Buache et de Mentelle ${ }^{48}$, ce sont des arguments méthodologiques qui sont cette fois mis en avant pour montrer l'incapacité des géographes à dégager des lois générales ou des rapports de causalité. L'auteur de l'article commence par montrer qu'à ces yeux un cours de géographie ne s'impose pas puisque les différentes branches de ce savoir peuvent respectivement être traitées dans les cours de mathématiques, d'histoire naturelle et d'histoire; puis il note :

«Si l'on voulait faire dans cette école un cours de géographie, il fallait s'y proposer, comme dans les autres cours, l'avancement de la science, le redressement des méthodes, et l'application de l'analyse à la géographie, qui peut y être soumise aussi bien que les autres sciences. Or, pour y parvenir, ce n'était pas, il nous semble, à des géographes de profession que l'on devait avoir recours : il est aisé d'en sentir les raisons, et par conséquent inutile de les dire. " ${ }^{49}$

Ces raisons tues par les animateurs de la Décade ont du mal à dissimuler le mépris de ces derniers pour l'ambition strictement descriptive que les « vrais " géographes donnent à leurs travaux. Si cela peut convenir aux ouvrages de vulgarisation, cela ne suffit pas pour conférer à la géographie une légitimité savante. En effet, les Idéologues n'admettent pas qu'un savant puisse ainsi se contenter de compiler les faits et exclure toute ambition explicative de ses questionnements. Cette critique de la "géographie des géographes » est exprimée de manière très explicite dans la Décade, mais en suggérant une autre manière de faire de la géographie, d'autres périodiques contribuent aussi au renouveau des savoirs géographiques.

III. Promouvoir la production de nouveaux savoirs géographiques

À partir de l'an III, plusieurs périodiques savants cherchent à promouvoir une géographie «régénérée » de la France. Un rédacteur de la Décade philosophique justifie ce renouvellement des savoirs géographiques en établissant une analogie entre la géographie et le bouleversement de la conception du territoire survenu pendant la Révolution. Il affirme de manière péremptoire :

«La nouvelle géographie de la France a été un des premiers bienfaits de la Révolution. La liberté ne pouvoit plus habiter ces anciennes provinces, honteuses barrières de la féodalité, vils apanages de nos tyrans. Cette révolution dans notre géographie devoit nous amener de nouvelles descriptions de la France. » ${ }^{50}$ 
nécessité d'une connaissance régénérée du territoire est déclinée à toutes les échelles, du national au local et de nombreux textes montrent qu'il n'y a aucune antinomie entre ces deux notions, bien au contraire. La Décade philosophique fait paraître en thermidor et fructidor an III une série de textes intitulés : « Le bourg et le hameau. Histoire extraite de la correspondance d'un des auteurs de la Décade " ${ }^{51}$. Ces textes sont présentés comme des lettres qui auraient été rédigées par un élève de l'École normale qui rentrait à pied dans son département, et qui aurait été adressées à un rédacteur du journal. Réelle ou fictive, cette correspondance insiste en tout cas sur l'accueil reçu par son auteur dans les villages ; les descriptions proposées sont censées susciter l'envie de parcourir le territoire pour en découvrir ses habitants. Désormais, aucun obstacle ni territorial, ni juridique, ni linguistique ne semble plus freiner la circulation des citoyens et leurs voyages doivent permettre d'accumuler des données sur les richesses des départements. Cette aspiration explique qu'entre 1789 et 1810, 157 voyages en France ont été publiés. Parmi eux, 45 concernent la préparation au voyage, plus d'une trentaine proposent un état des lieux et une douzaine rédigés par des ingénieurs s'attachent à l'aménagement du territoire. Plus de la moitié des voyages en France publiés à cette période entendent donc contribuer à cette géographie régénérée ${ }^{52}$. Dans les périodiques, plusieurs textes insistent sur l'importance du choix de l'échelle, sur la pertinence des descriptions régionales et de l'ancrage local de l'observateur dans ce renouveau de la géographie. Cette opinion est notamment défendue dans le compte rendu du Voyage dans la ci-devant Haute et Basse-Auvergne de Legrand :

«Aujourd'hui qu'il n'y a plus de provinces, que la République partagée en départemens à peu-près égaux, offre un ensemble plus régulier ; qu'une législation uniforme régit tous les Français ; que le mouvement continu d'une révolution de six années a fait disparaître une partie des différences de mœurs et d'usages, la simple curiosité sera peut-être moins excitée ; mais un plus grand intérêt, celui de connaître en détail le théâtre de tant d'événements ; celui de rechercher dans les mœurs nouvelles les traces des anciennes mœurs ; celui d'observer, après tant de peurs et de désastres, toutes les ressources de la culture, de l'industrie, du commerce d'un peuple qui peut nous placer au nombre de ses représentans, ou de ses premiers fonctionnaires ; en un mot, cet intérêt puissant, qui attache par tant de liens, l'homme libre à sa patrie, nous commandera même, après la paix, de négliger les pays étrangers et de parcourir, de visiter, d'étudier intimement le nôtre. » ${ }^{53}$

Trois numéros plus tard, le compte rendu se poursuit et donne de larges extraits de l'ouvrage en insistant fortement sur les ressources présentes dans ces départements et en précisant qu'en dépit de cette richesse « on n'a encore que des aperçus vagues sur

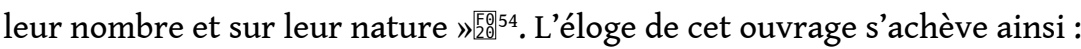

« Il faut terminer cet extrait, et renvoyer nos lecteurs à l'ouvrage : il doit intéresser tous les Français ; il doit leur faire regretter qu'il n'y en ait pas un pareil sur chacune de nos ci-devant provinces, et encourager les gens de lettres à qui leur position, ou quelque circonstance particulière, permet des voyages en France, à y porter les mêmes vues, pour en rapporter le même fruit. ${ }^{55}$

Si les périodiques encouragent les voyages des savants, ils s'efforcent aussi de mobiliser les habitants de chaque département pour qu'ils transmettent leurs observations et qu'ils contribuent ainsi au renouvellement de la géographie de la France. Dans le premier numéro des Annales de statistiques, les rédacteurs se demandent :

«Quel est le voyageur qui peut tout voir dans les lieux même qu'il parcourt? Et quel est celui qui peut parcourir en détail tous les départemens, toutes les 
communes, tous les villages, et jusqu'aux moindres établissements d'un état aussi vaste que la France ? Et même, en admettant cette possibilité, quel est l'homme, encore plus difficile à trouver, qui réunit l'immensité de connaissances locales absolument indispensables pour découvrir ce qui manque et ce qu'il convient de procurer à chaque département ? ${ }^{56}$

31 Le meilleur observateur est présenté comme celui qui connaît l'endroit qu'il décrit parce qu'il y vit. L'ancrage local des descriptions est regardée comme un gage de qualité : les rédacteurs des périodiques considèrent qu'un espace sera mieux décrit par quelqu'un qui le fréquente quotidiennement que par un voyageur de passage, si savant soit-il ${ }^{57}$. La mobilisation de tous les citoyens constitue donc une étape décisive dans la collecte des observations, mais aussi dans la vérification des faits ; elle est l'un des ressorts les plus efficaces du renouvellement des descriptions géographiques de la France. Le programme qui ouvre le premier numéro du Journal des mines suggère déjà ce type de collaboration ; il annonce en effet la mise en chantier d'une "description minéralogique de la France " et évoque la contribution de "savants estimables ", de "voyageurs employés à la recherche des mines", et de "ceux qui s'associeront volontairement à ces travaux $"{ }^{58}$. Dans l'» Apperçu [sic] de l'extraction et du commerce des substances minérales en France avant la Révolution ", le rédacteur invite «tous les citoyens à concourir avec zèle à l'exécution de ce travail ", il s'agit, en l'occurrence du "Tableau général des mines de France et de leurs produits " ${ }^{59}$. Ce type d'invitation se retrouve dans presque toutes les notices départementales. Comme l'écrit Jean-Claude Perrot, « l'amour de la petite patrie, les us et coutumes provinciales combattent pour le développement d'une science régionale " ${ }^{60}$.

Cette nécessaire mobilisation des citoyens suppose que ceux-ci soient formés. Plusieurs traités pédagogiques témoignent de cette préoccupation, mais les périodiques ne sont pas en reste. C'est en effet dans le Magasin encyclopédique que Jean-Denis Barbié publie sa « Nouvelle méthode pour enseigner la géographie » ${ }^{61}$. Dans ce texte, il insiste sur l'importance qu'il faut accorder au local dans la pédagogie et suggère d'appliquer l'analyse à la géographie. Pour cela, il recommande de partir du connu pour aller vers l'inconnu, c'est-à-dire de commencer par ce que les élèves ont sous les yeux pour terminer en leur expliquant le système du monde. Le géographe propose d'accompagner son enseignement d'outils spécifiques, il suggère de commencer par apprendre la notion d'échelle en observant la construction d'un plan, puis d'en venir à l'étude d'une carte topographique, d'une carte chorographique, d'une carte d'un pays, puis d'un continent, d'une mappemonde, enfin d'un globe. Cette progression reprend les trois échelles de la cartographie: la topographie qui permet l'étude d'un lieu particulier, la chorographie qui consiste à étudier une région ou un pays et la géographie qui se consacre à la description du globe. Cette manière de faire commencer l'enseignement de la géographie par le local montre que celle-ci est considérée comme mémoire locale ou mémoire par les lieux ${ }^{62}$. À première vue, cette méthode peut faire songer à celle préconisée par Mentelle dans sa Géographie enseignée par une méthode nouvelle, ou application de la synthèse à l'étude de la géographie. Dans cet ouvrage, Mentelle suggère en effet de partir du proche pour aller vers le lointain et il organise les leçons de géographie en fonction de cercles concentriques. Cependant, le point de départ reste toujours le même ; il s'agit en l'occurrence de Bourges, ville dont le géographe considère qu'elle est située à peu près au centre de la France, puis le cercle suivant englobe l'ensemble du département, puis un ensemble de sept départements..., etc. Comme l'a relevé Numa Broc, «l'ouvrage offre moins qu'il ne promettait et [...] la 
synthèse annoncée dans le titre fait cruellement défaut ${ }^{63}$. De plus, Mentelle part du proche pour aller vers le lointain ${ }^{64}$ alors que Barbié part de la connaissance du local spécifique à chaque élève- pour susciter l'intérêt pour la géographie. Cette position méthodologique de Barbié ne lui est pas propre, elle détermine aussi la conception du jardin géographique imaginé par Deleyre et présenté à la Convention en $1793{ }^{65}$. Elle traduit bien la forte emprise qui, pendant la Révolution, est conférée au local dans la formation d'une conscience géographique. Les périodiques savants se sont fait le relais de cette entreprise.

Ainsi, le Journal des arts et manufactures entend réunir des données et les transmettre au public avant que celles-ci ne soient combinées de manière plus systématique dans des ouvrages de synthèse. C'est en effet par l'accumulation d'observations locales, réunies dans des monographies que l'on entend parvenir à une vision globale du territoire. Dans le premier numéro, un avis adressé «aux correspondans sur la géographie industrielle » précise医:

«Lorsque tous les renseignements seront recueillis, on rédigera la géographie industrielle en un seul corps d'ouvrage ; on pourra même l'appuyer de cartes dans lesquelles on trouvera toutes les fabriques de la République. On doit présumer que les citoyens dont les pensées sont tournées vers l'économie politique, attacheront quelque prix à un pareil ouvrage, dans lequel ils pourront trouver une réunion de données numériques qui a manqué jusqu'ici à cette science. " ${ }^{66}$

Non seulement les périodiques savants suscitent l'accumulation d'informations géographiques dans le cadre d'enquêtes conduites à l'échelle locale, mais ils proposent aussi une manière de les ordonner. Ainsi, dans le premier numéro des Annales de statistiques le géographe Mentelle publie une description de la France censées servir de modèle. Il commence par caractériser l'étendue du territoire de la France, puis il décrit la «distribution physique» en reprenant le cadre alors classique et commode des bassins fluviaux et des chaînes de montagne, il termine sa contribution par une "Division des départements par bassins de grandes rivières ". Ce souci de replacer les données dans un cadre descriptif homogène est nécessaire à l'accumulation de données et Mentelle opte pour un ordre d'exposition «naturel » :

«Nous croirons suivre une marche plus naturelle, en établissant la division de la France d'après des bases physiques, telles que la direction des montagnes ou des terrains élevés et telles encore que les bassins désignés par les noms des fleuves qui en coupent la partie la plus profonde. ${ }^{67}$

35 À l'image des préoccupations exprimées autour du projet statistique animé par Chaptal, on retrouve dans les périodiques savants la volonté de fixer un ordre d'exposition pertinent et stable ${ }^{68}$. Dans le Journal des Mines, les données minéralogiques recueillies sont exposées en suivant le maillage départemental et c'est l'ordre alphabétique qui régit l'agencement des notices publiées. Contrairement au Journal des Mines, les Annales de statistiques font paraître leurs descriptions départementales en suivant le découpage des bassins fluviaux. Bien que les principes d'exposition diffèrent d'un périodique à l'autre, le savoir géographique est dans les deux cas ordonné pour mieux servir de la connaissance du territoire et améliorer sa mise en valeur. Ces imprimés confirment les analyses de Dominique Margairaz qui montrent que pendant la Révolution les savoirs de l'espace ne relèvent plus de la curiosité, mais de l'exploitation rationnelle des ressources; ils ne se contentent plus de la nomenclature des lieux mais exigent des descriptions de qualité ${ }^{69}$. Certains périodiques savants proches des rouages du ministère de l'Intérieur s'arrogent même un rôle notable dans cette production 
renouvelée du savoir géographique : non seulement ils suscitent la collecte des faits, mais ils proposent aussi des méthodes pour exposer ces données.

En contribuant à la diffusion des savoirs de l'espace, les périodiques savants de la Révolution participent à la formation d'une conscience géographique nationale. La géographie n'est plus seulement regardée comme une science aimable, elle apparaît dans leurs pages comme une science utile. Ainsi, contrairement à la presse savante qui, sous l'Ancien Régime, conférait une large place aux espaces lointains ${ }^{70}$, ce sont désormais les départements français qui retiennent prioritairement l'attention des rédacteurs. Le territoire français doit en effet être connu et décrit pour être maitrisé : telle est la mission assignée à la géographie. Certains périodiques ne se contentent pas de refléter cette évolution, ils accompagnent ce processus et quelques uns contribuent même à activer la production de savoirs géographiques. Cependant plus que la géographie, ce sont les techniques descriptives et les sciences connexes (statistique et géologie) qui bénéficient de ce renouveau.

\section{NOTES}

1.Sur le statut de la géographie, voir Hélène BLAIS et Isabelle LABOULAIS-LESAGE [dir.], Géographies plurielles. Les sciences géographiques au moment de l'émergence des sciences humaines (1750-1850), à paraître chez L'Harmattan, dans la collection Histoire des Sciences de l'homme, début 2005.

2.En 1795, lors de la fondation de l'Institut national, la géographie échoit à la classe des sciences morales et politique, et, jusqu'en 1803, six géographes animent les travaux de cette section. Lorsqu'elle est supprimée par Napoléon, trois d'entre eux sont affectés à la section " géographie et navigation » de la classe des sciences physiques et mathématiques, et les trois autres sont replacés dans la troisième classe consacrée à l'histoire et aux langues anciennes. Les tiraillements de la géographie entre sciences et belles lettres refont donc surface. Sur cette question, on se reportera à Hélène Blais, «La géographie académique entre sciences et belles lettres (1795-1832), in Hélène BLAIS et Isabelle LABOULAIS-LESAGE [dir.], Géographies plurielles, op.cit.

3.Daniel RAICHVARG, Jean JACQUES, Savants et Ignorants. Une histoire de la vulgarisation des sciences, Paris, Seuil, 1991, 291 p.

4.Daniel NORDMAN, « Une représentation géographique du territoire sous la Révolution: le "jardin patriotique" ", Lectures, 24, 1989/1, Bari, pp. 223-240 ; idem, « La pédagogie du territoire, 1793-1814 », in Daniel NORDMAN et Marie-Vic OzOUF-MARIGNIER [dir.], Le territoire (1). Réalités et représentations, Paris, E.H.E.S.S., 1989, pp. 62-64 ; Jean-Marc BESSE, Face au monde. Atlas, jardins, géoramas, Paris, Desclée de Brouwer, 2003, 243 p.

5.Nous indiquons entre parenthèses ici les périodes pour lesquelles chaque titre a été dépouillé : le Bulletin de littérature, des sciences et des arts (an IV - an V), le Bulletin des sciences de la Société philomathique de Paris (1791 - 1805), la Décade philosophique littéraire et politique (an II - an VI), le Magasin encyclopédique ou Journal des sciences, des lettres et des arts (an III - an VII), le Journal des Mines (an III - an X), le Journal des Arts et manufactures 
(1801), et les Annales de statistique ou Journal général d'économie politique (...), de géographie (an X). Toutes les citations reproduites dans cet article respectent l'orthographe originale.

6.Marie-Noëlle BOURGUET, Déchiffrer la France. La statistique de la France à l'époque napoléonienne, Paris, Éd. des Archives Contemporaines, 1988 ; Marie-Vic OzOUF-MARIGNIER, La formation des départements. La représentation du territoire français à la fin du XVIIIe siècle, Paris, E.H.E.S.S., 1992 ; Jean-Claude PERROT, L'âge d'or de la statistique régionale française (an IV-1804), Paris, Société des Études robespierristes, 1977 ; Dominique MARGAIRAZ, Du lieu commun à l'esprit public. François de Neufchâteau. Essai de biographie intellectuelle, Thèse d'État, Paris I, 2001, à paraître aux Publications de la Sorbonne.

7.Jean-Pierre VITTU, Le Journal des savants et la République des lettres, 1664-1714, Thèse de doctorat, Université de Paris I, 1998 ; « Périodiques », in Michel BLAY et Robert HALLEUX, La science classique, XVI-XVIIIe siècles, Paris, 1998, pp. 140-148 ; idem, «Qu'est-ce qu'un article au Journal des savants de 1665 à 1714 ? », Revue française d'histoire du livre, $\mathrm{n}^{\circ}$ 112-113, 2001, pp. 129-148.

8.«Sur une projection proposée par feu M. Lorgna », Bulletin des sciences de la société philomathique, $\mathrm{n}^{\circ} 29$, thermidor an VII, pp. 37-39; « Observations faites par le Citoyen Girod-Chantrans pendant un voyage dans les Basses Pyrénées et en Catalogne », ibid., n 32, brumaire an VIII, pp. 60-63; « Extrait d'une lettre de M. Humboldt, renfermant des observations géographiques et physiques ", ibid., $\mathrm{n}^{\circ} 49$, germinal an IX, pp. 4-6 ; «Extrait d'un Mémoire de C. Coquebert-Montbret, sur des cartes manuscrites dressées dans la première moitié du 16e siècle ", ibid., nº 81, frimaire an XII, pp. 163-164 + planche.

9.Décade philosophique, vol. XIV, $\mathrm{n}^{\circ}$ 15, 30 pluviôse an VI, pp. 321-331, particulièrement p. 322.

10.« Notes sur la Nouvelle-Guinée par Barbié du Bocage », Magasin encyclopédique, tome premier, an III, pp. 526 ; « Nouvelle méthode pour enseigner la géographie par J.D. Barbié », ibid., tome second, an III, pp. 483-491 ; « Lettre de J.D. Barbié, aux rédacteurs du Magasin encyclopédique sur la terre de Kerguelen », ibid., p. 205 ; « Découverte de vicus Helena de Sidoine Apollinaire et du Clausula de Baudri et de Sigebert », ibid., troisième année, tome troisième, 1797, p. 162 ; « Des peuples connus dans l'antiquité sous le nom de Boréens, Hyperboréens, etc. ", ibid., quatrième année, tome cinquième, 1799 , p. 196 ; « Lettre de C. Soreau sur les différentes hauteurs des villes et des montagnes les plus connues ", ibid., cinquième année, tome quatrième, an VIII, p. 385. 11.Jean-Baptiste DELAMBRE, Rapport historique sur les progrès des sciences mathématiques depuis 1789, 1810, p. 28, cité dans Numa BROC, La géographie des philosophes. Géographes et voyageurs français au XVIIIe siècle, Paris, Ophrys, 1975, p. 460.

12.Michèle DUCHET, Anthropologie et histoire au siècle des Lumières [1971], postface de Claude BLANCKAERT, Paris, Albin Michel, 1995, 611 p.

13. « Extrait d'une correspondance manuscrite de Mirabeau contenant la description de son arrivée en Angleterre ", Décade philosophique, vol. VII, n 57, 30 brumaire an IV, pp. 347-352; « Fragment d'un voyage de la Nort-Hollande, extrait du journal manuscrit des voyages du citoyen Thouin, dans la Belgique et la Hollande ", ibid., vol. VIII, nº 63, 30 nivôse an IV, pp. 142-148 ; « Extrait d'une lettre du citoyen Monge commissaire du gouvernement en Italie », ibid., vol. X, n 85,10 fructidor an IV, pp. 406-408. 14.Décade philosophique, vol. IX, $\mathrm{n}^{\circ}$ 76, 10 prairial an IV, pp. 398-412 et vol. IX, $\mathrm{n}^{\circ}$ 77, 20 prairial an IV, pp. 454-464; Magasin encyclopédique, seconde année, tome second, an IV, pp. 70-103. 
15.Hélène BLAIS, « Le rôle de l'Académie des sciences dans les voyages d'exploration au XIXe siècle ", La revue pour l'histoire du C.N.R.S., n 10 ; mai 2004 ; pp. 34-43.

16.Annales de statistiques, t. Ier, 1802 (an X), pp. XLVI-XLVII.

17.Journal des Mines, vol. VI, $\mathrm{n}^{\circ}$ 32, floréal an V, pp. 619-620.

18.Cité dans Daniel NORDMAN, Marie-Vic OZOUf-MARIGNIER (dir.), Le territoire (1), op. cit., p. 64.

19.Journal des arts et manufactures, $n^{\circ} 1$, an III, p. 49.

20.Jean-Claude PERROT, op. cit., p. 18.

21.Anne GODLEWSKA, Geography unbound. French Geographic Science from Cassini to Humboldt, Chicago, The University of Chicago Press, 1999, pp. 193-232 et pp. 235-265.

22.Décade philosophique, vol. II, $\mathrm{n}^{\circ} 10,10$ thermidor an II, p. 21

23.Ibid., vol. IX, $\mathrm{n}^{\circ}$ 74, 20 floréal an IV, troisième de couverture.

24.Magasin encyclopédique, $\mathrm{n}^{\circ}$ 1, troisième année, premier tome, 1797 (an V), p. 260.

25.Denis REYNAUD, « Journalisme d'Ancien Régime et vulgarisation scientifique », in Lise ANDRIES (dir.), Le partage des savoirs. XVIII-XIXe siècles, Collection « littérature \& idéologies », Lyon, Presses universitaires de Lyon, 2003, pp. 121-134, et plus particulièrement sur la question des extraits, pp. 129-130.

26.Yasmine MARCIL, «Les normes d'écriture du récit de voyage dans la presse périodique de la fin du XVIIIe siècle ", Seuils \& Traverses, Enjeux de l'écriture du voyage, (Actes du colloque de Brest, 6-8 juillet 2000), textes réunis par J.-Y. LE DISEZ et Jan BORN, Université de Bretagne occidentale, Université de Versailles Saint-Quentin-en-Yvelines, 2002, vol. 2, pp.249-260.

27.Compte rendu du Voyage chez différentes nations sauvages de l'Amérique septentrionale, renfermant des détails curieux sur les mours, usages, cérémonies religieuses, le système militaire, etc., des Cahnuugas, des Indiens des cinq et six nations, Mohawks, Connecedagas, Iroquois, des Indiens Chippeways et autres sauvages de diverses tribus, par J. Long, trafiquant et interprète de langues indiennes, traduits de l'anglois avec des notes intéressantes par Billecocq, citoyen françois, à Paris, chez Prault l'aîné. Compte rendu publié dans la Décade philosophique, vol. I, $\mathrm{n}^{\circ} 8,20$ messidor an II, pp. 478-484, et plus particulièrement p. 479.

28. Bulletin de la littérature, des sciences et des arts, vol. I, n 1, s.d., p. 2.

29.Ibid., p. 484.

30.Décade philosophique, vol. XI, $\mathrm{n}^{\circ}$ 5, 20 brumaire an V, pp. 284-291.

31.Ibid., vol. XI, $\mathrm{n}^{\circ}$ 6, 30 brumaire an V, p. 356 .

32.Voyage en Angleterre, en Ecosse et aux îles Hébrides, ayant pour objet les sciences, les arts, l'histoire naturelle et les mœurs par B. Faujas de Saint-Fond, 2 vol., in- $8^{\circ}$, Jansen. Compte rendu publié dans le Magasin encyclopédique, 1797 (an V), n 4, pp. 21-35.

33. Voyage philosophique et pittoresque sur les rives du Rhin, à Liège, dans la Flandre, la Brabant, la Hollande, etc. fait en 1790 par Georg Forster, l'un des compagnons de Cook, traduit de l'allemand avec des notes critiques sur la physique, la politique et les arts, par Charles Pougens, 2 vol., in- $8^{\circ}$, à Paris, chez Buisson. Compte rendu publié dans la Décade philosophique, vol. III, $\mathrm{n}^{\circ} 20,20$ brumaire an III, pp. 278-288, plus particulièrement $\mathrm{p}$. 280.

34.Compte rendu par Coquebert de Geographiske chartor osver Svenge, etc. Cartes géographiques de la Suède, accompagnées de vues dessinées d'après nature; le tout publié par le baron S.G. Hermelin, conseiller les mines, Stockholm, 1797, format atlas, in Magasin encyclopédique, 1798 (an VI), n 3, p. 208. 
35.Ibid.

36. Carte de la France où l'on a essayé d'exprimer les configurations de son territoire, par une nouvelle méthode de nivellement, par l'ingénieur géographe Dupain-Triel, chez l'auteur ; compte rendu dans le Magasin encyclopédique, 1799 (an VII), nº 3, p. 422.

37. Bulletin de la littérature, des sciences et des arts, vol. I, n 30, s.d., p. 236.

38. Le département du Mont-Blanc, réuni à la République française, divisé en 7 districts et 83 cantons, gradués d'après la division décimale du quart du méridien par le citoyen JeanBaptiste Raymond, ingénieur-géographe. Compte rendu publié dans le Bulletin de la littérature, des sciences et des arts, vol. I, n 3, s.d., p. 23.

39.Isabelle LABOULAIS-LESAGE, « De la mise en ordre du territoire : le recours au discours géographique pour cautionner les nouveaux découpages territoriaux ", in Jean-Pierre JESSENNE (dir.), Du Directoire au Consulat, 3. Brumaire dans l'histoire du lien politique et de l'Etat-Nation, Centre de Recherche sur l'Histoire de l'Europe du Nord-Ouest, Lille, 2001, pp. 561-573.

40.Magasin encyclopédique, 1799 (an VIII), n5, p. 563.

41.Décade philosophique, vol. I, $\mathrm{n}^{\circ}$ 9, 30 messidor an II, pp. 522-523.

42.Ibid., vol. IV, $\mathrm{n}^{\circ}$ 30, 30 pluviôse an III, pp. 337-338.

43. Décade philosophique, vol. IV, $\mathrm{n}^{\circ}$ 30, 30 pluviôse an III, pp. 343.

44.Bulletin de la littérature, des sciences et des arts, vol. I, n 15, s.d., pp. 115-116.

45.Géographie de France, suivant la division en 88 départemens contenant des détails sur l'origine, les révolutions, l'état actuel, les productions, l'industrie, le commerce, les édifices des différens âges et de différens genres, les promenades, places publiques, statues, inscriptions ; les anecdotes et singularités historiques de chaque ville; le caractère et les ouvrages des hommes célèbres ; les costumes frappans, etc. 4 vol. in-12. Chez Devaux. Compte-rendu publié dans la Décade philosophique, vol. IV, $\mathrm{n}^{\circ}$ 30, 30 pluviôse an III, pp. 337-344.

46.Mona Ozouf, « Voyages en France dans la décennie révolutionnaire », in W. FRIJHOFF, et $\mathrm{R}$. DEKKER (dir.), Le voyage révolutionnaire. Actes du colloque franco-néerlandais $d u$ Bicentenaire de la Révolution française, Amsterdam, 12-13 octobre 1989, Rotterdam, Erasmus Universiteit, 1991, pp. 15-35.

47. Voyage dans les départements de la France, enrichis de tableaux géographiques et d'estampes, par les citoyens Lavallée pour le texte ; Brion père pour la partie géographique et Brion fils pour le dessin. Compte-rendu publié dans Bulletin de la littérature, des sciences et des arts, Vol. I, n 9, s.d., pp. 68-69.

48.Daniel noRdMAN (dir.), L'École normale de l'an III. Leçons d'histoire de géographie d'économie politique, Paris, Dunod, 1994, pp. 137-161 ; Anne GODLEWSKA, op. cit., pp. 62-65. 49.Décade philosophique, vol. IV, $\mathrm{n}^{\circ}$ 30, 30 pluviôse an III, p. 352.

50.Décade philosophique, vol. I, $\mathrm{n}^{\circ}$ 9, 30 messidor an II, p. 522.

51.Ibid., vol. VI, $\mathrm{n}^{\circ} 48,30$ thermidor an III, pp. 340-361; ibid., vol. VI, $\mathrm{n}^{\circ} 49,10$ fructidor an III, pp. 410-421 ; ibid., vol. VI, n' 50, 20 fructidor an III, pp. 471-478 ; ibid., vol. VI, $\mathrm{n}^{\circ} 51,30$ fructidor an III, pp. 541-549.

52.Daniel NORDMAN, Marie-Vic OZOUF-MARIGNIER (dir.), Le territoire (1), op. cit., p. 72.

53.Voyage fait en 1787 et 1788, dans la ci-devant Haute et Basse-Auvergne, aujourd'hui départemens du Puy-de-Dôme, du Cantal et partie de celui de la Haute-Loire. Par Legrand, 3 vol., in- $8^{\circ}$, à Paris. Compte rendu publié dans la Décade philosophique, vol. V, $\mathrm{n}^{\circ} 36,30$ germinal an III, pp. 145-146.

54.La Décade philosophique, vol. V, n³9, 30 floréal an III, p. 334.

55.Ibid., pp. 346-347.

56.Annales de statistiques, t. Ier, 1802 (an X), p. XLVIII. 
57.« Le savoir géographique s'apparente alors à un savoir quotidien, savoir des villes et savoir des champs, qui s'acquiert dans la fréquentation des choses et des êtres, fréquentation d'abord visuelle, un savoir à l'estime pourrait-on dire, qui s'exerce sans livres ni cartes. Ce savoir, qui se présente comme un jugement de l'œil, est un savoir des proximités et des distances, qui s'effectue pas l'intermédiaire d'une discrimination active des places. Savoir, par conséquent des distributions, des distinctions, des relations, mais qui se joue silencieusement, à un niveau qui précède tout parole, et qui se présente comme une mise en espace quasiment physique de la consciences ", JeanMarc BESSE, op. cit., p. 160.

58. Charles Coquebert, « Programme », Journal des Mines de la République, $\mathrm{n}^{\circ} 1$, vendémiaire an III, p. 8.

59.« Apperçu [sic] de l'extraction et du commerce des substances minérales en France avant la Révolution », Journal des Mines, $\mathrm{n}^{\circ}$ 1, vendémiaire an III, p. 56.

60.Jean-Claude PERROT, op. cit., p. 66.

61.Magasin encyclopédique, $\mathrm{n}^{\circ}$ 2, 1795, an III, pp. 483-491.

62.Jean-Marc BESSE, op. cit., p. 130.

63.Numa BROC, op. cit., p. 470.

64.À ce titre, il est surprenant de lire dans le compte rendu de La Géographie enseignée par une méthode nouvelle, ou application de la Synthèse à l'étude de la géographie, ouvrage destiné aux Écoles primaires, avec neuf cartes enluminées, par le citoyen Mentelle, compte rendu publié dans le Magasin encyclopédique le commentaire suivant : « Cet ouvrage, ainsi qu'il l'avance, avoit été composé par ordre du comité d'instruction publique. Il est rédigé d'après une méthode nouvelle que le citoyen Barbié-du-Bocage a déjà recommandé dans un de nos numéros, et qui a été mise en usage par les auteurs du Porte-Feuilles des Enfants, c'est de ne parler d'abord aux enfants que des lieux qu'ils habitent, et de les faire partir de ce point pour connoître les autres lieux. L'ouvrage est accompagné de petites cartes exécutées d'après ce principe : il peut être très utile pour l'enseignement de la géographie ", Magasin encyclopédique, 1796 (an IV), n 2, p. 136. L'auteur cherche probablement à rappeler le bien-fondé de la méthode de Barbié et pour cela, il déforme celle préconisée par Mentelle.

65.Jean-Marc BESSE, op. cit., p. 99 et p. 105.

66.Journal des arts et manufactures, $\mathrm{n}^{\circ} 1$, an III, p. 55.

67.Annales de statistiques, t. 1er (an X), pp. 62-63.

68. Marie-Noëlle BOURGUET, Déchiffrer la France, op. cit.

69.Dominique MARGAIRAZ, « La géographie des administrateurs ", in Hélène BLAIS, Isabelle LABOULAIS-LESAGE (dir.), Géographies plurielles, op. cit.

70.Yasmine MARCIL, Récits de voyage et presse périodique au XVIIIe siècle : de l'extrait à la critique, Thèse de doctorat soutenue sous la direction de Roger Chartier, E.H.E.S.S., 2000.

\section{RÉSUMÉS}

Pendant la Révolution française, les savoirs géographiques sont de moins en moins considérés comme relevant d'une culture de curiosité; au contraire leur utilité pour le citoyen et pour 
l'administrateur est affirmée avec force. Les périodiques savants - généralistes ou spécialisés accompagnent cette évolution, à la fois en contribuant à la diffusion des savoirs géographiques, en formulant un certain nombre de critiques à l'égard de textes qui n'ont pas la rigueur désormais requise par ce domaine de savoir, mais aussi en incitant savants et citoyens à produire de nouvelles géographies ou du moins à contribuer à la réunion des données nécessaires à l'élaboration de nouvelles descriptions.

The Role of Learned Journals in the Spread of Geographical Knowledge during the French Revolution. During the French Revolution, a knowledge of geography was less and less considered a matter relating to a culture of curiosity and came to be forcefully proclaimed as a matter of utility for the citizen and administrator. Learned journals, whether of general or specialized interest, featured prominently in this trend by contributing to the spread of geographical knowledge, by voicing criticism of texts deemed to lack the scientific rigour now required in this field, and also by urging scientists and citizens to produce new geographies or at least help gather the data required for the elaboration of new descriptions.

INDEX

Mots-clés : géographie, récits de voyages, périodiques savants, description

\section{AUTEUR}

ISABELLE LABOULAIS-LESAGE

Université Marc Bloch - Strasbourg 2, 10 place Saint-Etienne 67000 Strasbourg 